\title{
Utility and safety of depth electrodes within the supratemporal plane for intracranial EEG
}

\author{
Yasunori Nagahama, MD, ${ }^{1}$ Alan J. Schmitt, MD, ${ }^{2}$ Brian J. Dlouhy, MD, ${ }^{1,3}$ Adam S. Vesole, BS, ${ }^{4}$ \\ Phillip E. Gander, PhD, ${ }^{1}$ Christopher K. Kovach, PhD, ${ }^{1}$ Daichi Nakagawa, MD, PhD, ${ }^{1}$ \\ Mark A. Granner, MD, ${ }^{2}$ Matthew A. Howard III, MD, ${ }^{1,3}$ and Hiroto Kawasaki, MD1
}

\begin{abstract}
Departments of ${ }^{1}$ Neurosurgery and ${ }^{2}$ Neurology, University of lowa Hospitals and Clinics; ${ }^{3}$ Pappajohn Biomedical Institute, University of lowa Carver College of Medicine; and ${ }^{4}$ Carver College of Medicine, University of lowa Hospitals and Clinics, lowa City, lowa
\end{abstract}

\begin{abstract}
OBJECTIVE The epileptogenic zones in some patients with temporal lobe epilepsy (TLE) involve regions outside the typical extent of anterior temporal lobectomy (i.e., "temporal plus epilepsy"), including portions of the supratemporal plane (STP). Failure to identify this subset of patients and adjust the surgical plan accordingly results in suboptimum surgical outcomes. There are unique technical challenges associated with obtaining recordings from the STP. The authors sought to examine the clinical utility and safety of placing depth electrodes within the STP in patients with TLE.

METHODS This study is a retrospective review and analysis of all cases in which patients underwent intracranial electroencephalography (iEEG) with use of at least one STP depth electrode over the 10 years from January 2006 through December 2015 at University of lowa Hospitals and Clinics. Basic clinical information was collected, including the presence of ictal auditory symptoms, electrode coverage, monitoring results, resection extent, outcomes, and complications. Additionally, cases in which the temporal lobe was primarily or secondarily involved in seizure onset and propagation were categorized based upon how rapidly epileptic activity was observed within the STP following seizure onsets: within 1 second, between 1 and 15 seconds, after 15 seconds, and not involved.
\end{abstract}

RESULTS Fifty-two patients underwent iEEG with STP coverage, with 1 STP electrode used in $45(86.5 \%)$ cases and 2 STP electrodes in the other cases. There were no complications related to STP electrode placement. Of 42 cases in which the temporal lobe was primarily or secondarily involved, seizure activity was recorded from the STP in 36 cases (85.7\%): in 5 cases (11.9\%) within 1 second, in 5 (11.9\%) between 1 and 15 seconds, and in 26 (61.9\%) more than 15 seconds following seizure onset. Seizure outcomes inversely correlated with rapid ictal involvement of the STP (Engel class I achieved in $25 \%, 67 \%$, and $82 \%$ of patients in the above categories, respectively). All patients without ictal STP involvement achieved seizure freedom. Only 4 (11.1\%) patients with STP ictal involvement reported auditory symptoms.

CONCLUSIONS Ictal involvement of the STP is common even in the absence of auditory symptoms and can be effectively detected by the STP electrodes. These electrodes are safe to implant and provide useful prognostic information. https://thejns.org/doi/abs/10.3171/2018.4.JNS171812

KEYWORDS invasive electroencephalography; outcome; planum temporale; temporal lobe epilepsy; temporal operculum; temporal plus epilepsy

$\mathrm{T}$ EMPORal lobe epilepsy (TLE) is a common form of focal epilepsy, and in many patients with TLE, seizures are refractory to medical management. Seizure focus resection surgery can be highly effective in this group of patients. ${ }^{15}$ Different subtypes of TLE have been recognized, based on different locations of seizure onset zones (i.e., mesial or limbic, lateral or neocortical, and me- siolateral). ${ }^{8}$ In any individual patient, there may be multiple areas of high epileptogenicity that may collectively constitute an epileptogenic network. ${ }^{2}$ In some cases, the epileptogenic zone may extend beyond the typical boundary of the anterior temporal lobectomy (ATL) to include the neighboring peri-sylvian areas, such as the orbitofrontal cortex, the supra-sylvian operculum, the insula, and

ABBREVIATIONS ATL = anterior temporal lobectomy; $A T O=$ anterior temporal operculum; EEG = electroencephalography; HG = Heschl's gyrus; iEEG = intracranial EEG; IN = insula; PT = planum temporale; SEEG = stereo-EEG; STP = supratemporal plane; TLE = temporal lobe epilepsy.

SUBMITTED July 24, 2017. ACCEPTED April 2, 2018.

INCLUDE WHEN CITING Published online September 7, 2018; DOI: 10.3171/2018.4.JNS171812. 
the temporoparietooccipital junction (i.e., "temporal plus epilepsy").,2,12 These neighboring areas may not be recognized as part of the epileptogenic network in the course of a patient's pre-resection evaluation; failure to fully delineate this network and incorporate this information into the surgical treatment plan may contribute to suboptimal TLE surgery outcomes..$^{1,12}$

In complex TLE cases in which the results of noninvasive studies are inconclusive, invasive extraoperative intracranial electroencephalography (iEEG) monitoring provides essential information to guide epilepsy surgery decision-making. Although the mesial limbic temporal structures and the anterior, lateral, and ventral neocortical areas are readily accessible using depth and subdural surface electrodes, the dorsal temporal cortex (i.e., supratemporal plane [STP]) presents special technical challenges for electrode coverage. The dorsal temporal cortex forms the ventral bank of the sylvian fissure and immediately abuts the branches of the middle cerebral artery and peri-sylvian veins. One well-established method for obtaining electrode coverage in this area involves use of stereo-electroencephalography (SEEG).,16 Orthogonally placed depth electrodes inserted through the temporal operculum into the insula have been utilized in SEEG in suspected TLE with insular involvement., 5 The SEEG approach enables image-guided stereotactic placement of multiple depth electrodes via electrode trajectories that do not intersect with identified vascular structures. Our group has adopted a modified approach whereby depth electrodes are placed within the STP as part of a craniotomy procedure. ${ }^{10,11}$ We routinely place at least 1 STP depth electrode along Heschl's gyrus (HG) in cases of suspected TLE where iEEG is indicated, regardless of the presence of auditory symptoms. A diagonal insertion trajectory is used that avoids traversing sulci or blood vessels, while enabling sampling of anterolateral and posteromedial portions of the STP with 1 multicontact electrode. In some cases, depth electrodes are placed within the anterior temporal operculum (ATO) and planum temporale (PT), with tip contacts terminating in the anterior and posterior insula (IN), respectively.

In this study, we performed a retrospective review and analysis of all cases in which patients underwent iEEG involving use of at least 1 depth electrode in the STP over the 10 years from January 2006 through December 2015 at the University of Iowa Hospitals and Clinics. We aimed to evaluate the clinical utility and safety of the STP depth electrodes in suspected TLE cases.

\section{Methods}

\section{Patient Population and Surgical Method}

All adult and pediatric patients who underwent extraoperative iEEG involving use of at least 1 depth electrode within the STP between January 2006 and December 2015 at University of Iowa Hospitals and Clinics were identified from a prospectively maintained epilepsy surgery database. The database included a total of 52 such patients, and all of these patients underwent craniotomy for electrode placement. The current study was approved by the University of Iowa Institutional Review Board.
All the cases were individually discussed at the multidisciplinary epilepsy conference, involving epileptologists, neuroradiologists, neuropsychologists, and neurosurgeons. Patients suspected to have medically intractable epilepsy with seizure onset or early propagation possibly involving the temporal lobe and preoperative epilepsy workup data that were not concordant for unilateral mesial temporal seizure onset were determined to be candidates for videoEEG monitoring with intracranial electrodes. The lateral and ventral temporal cortex were typically covered with grid and strip electrodes and the mesial temporal lobe was sampled with depth electrodes. Other lobes were also covered as suggested by preoperative seizure workup data. The average total number of electrode contacts was $176 \pm$ 54 (mean \pm SD). The number of electrode contacts among patients grouped by STP seizure involvement was not significantly different, with $127 \pm 66,184 \pm 67,182 \pm 50$, and $186 \pm 40$ for $0-1$ second, $1-15$ seconds, $>15$ seconds, and no involvement groups, respectively (1-way ANOVA, F = $1.69, \mathrm{p}=0.185$ ).

We covered the STP with 1 or a combination of depth electrodes placed along HG, through the ATO into the anterior IN, and/or within the PT terminating in the posterior IN.

The technical details of the HG depth electrode stereotactic implantation method have been reported previously. ${ }^{10,11}$ Briefly, the HG runs obliquely from posteromedial to anterolateral orientation within the STP. The anterolateral and posteromedial margins of the HG were chosen as an entry point and a target point, respectively, for stereotactic placement of the HG depth electrode (Figs. 1 and 2). The depth of the HG electrode typically measured about $40-50 \mathrm{~mm}$. The anterior peri-sylvian temporal cortex is exposed during craniotomy to enable the unique oblique orientation of the HG electrode placement. The other STP electrodes were inserted through the ATO and the PT and directed perpendicular to the lateral temporal cortical surface to target the anterior and posterior IN, respectively.

Although the lateral (neocortical) subtype of TLE has been more frequently associated with auditory symptoms than have the mesial (limbic) or mesial-lateral subtypes, ${ }^{8}$ this association is not always present. Therefore, a history of auditory symptoms was not a prerequisite for placement of STP electrodes, given that the absence of auditory symptoms does not rule out involvement of the STP in a patient's seizure disorder.

When a seizure focus was noted to involve areas near the eloquent areas (i.e., language areas, primary motor and somatosensory areas), electrical stimulation mapping was performed to help guide resection planning. Electrical stimulation was not used for the purpose of determining a seizure onset zone by eliciting ictal symptoms. Therefore, regardless of ictal involvement of the STP, the electrodes placed in the STP were not used for electrical stimulation mapping.

In most of the cases, resective surgery consisted of resection of the amygdala and hippocampus and disconnection of the temporal pole. In some cases, resection also included the orbitofrontal cortex, posterior lateral temporal cortex, and insula, as guided by iEEG results. For 3 patients who had complete resection of STP seizure sites, 

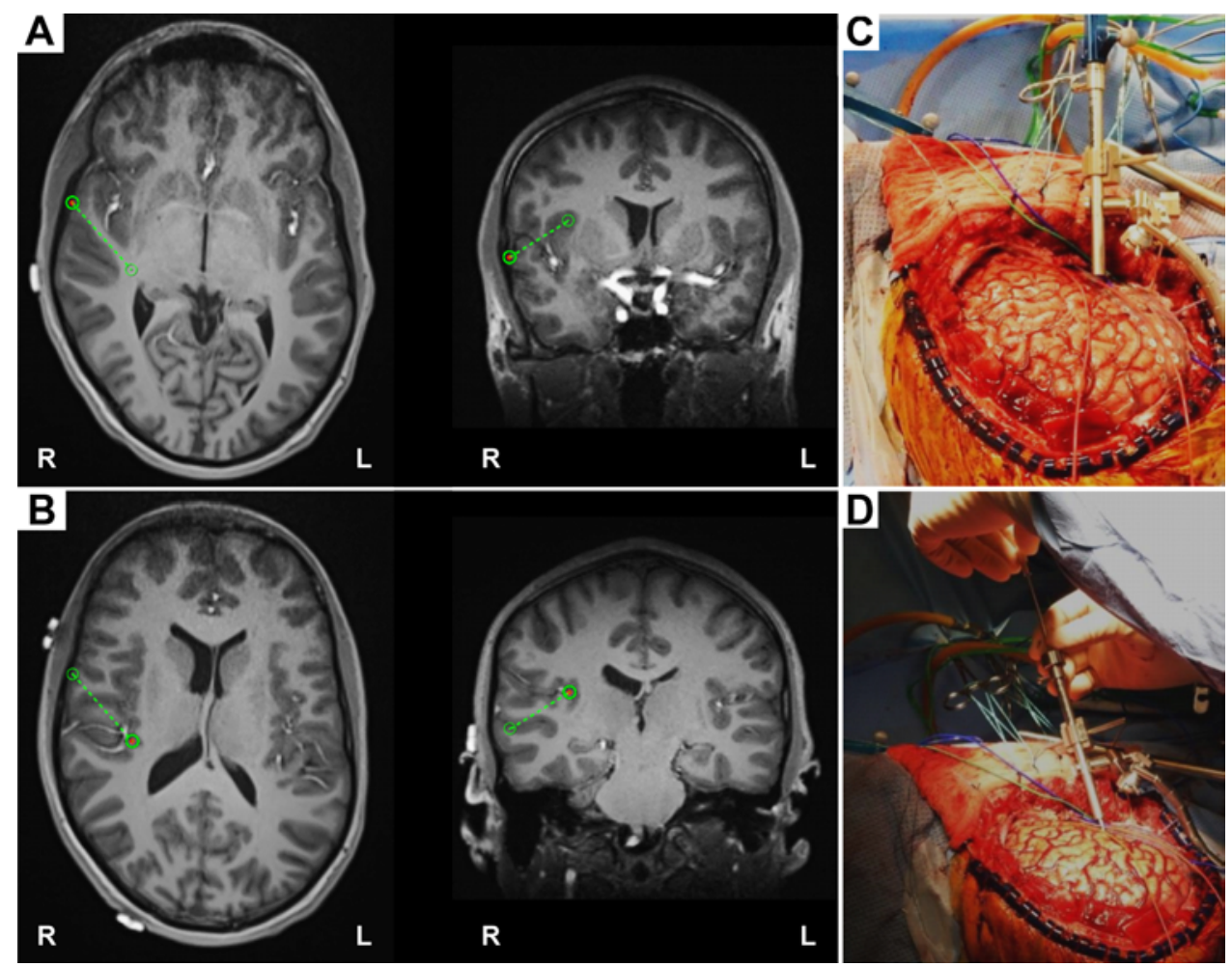

FIG. 1. Intraoperative placement of HG depth electrode. A: Planning view on the frameless stereotactic system (Stealth Navigation, Medtronic) showing the entry point and the trajectory (green circles and dotted lines). B: Similar planning view showing the target and the trajectory. C and D: Intraoperative photographs showing placement of the HG depth electrode. A Stealth Navigus probe was used to select the appropriate trajectory of a guiding tube positioned over the entry point $(\mathrm{C})$. An electrode-guiding cannula was advanced through the tube to the previously determined depth (D). An actual depth electrode was subsequently passed through the cannula, followed by removal of the guiding tube/cannula system. Note the unique anterolateral-to-posteromedial trajectory within the STP for placement of the HG depth electrode. Reproduced from Nagahama Y, et al: Localization of musicogenic epilepsy to Heschl's gyrus and superior temporal plane: case report. J Neurosurg 129:157-164, 2018. Published with permission.

Figure is available in color online only.

completeness of STP resection was confirmed with MRI in 2 cases and with axial and coronal CT scan in the other case. For 2 patients who had partial resection of STP seizure sites (one with seizure onset in the STP and the other one with early [1-15 seconds] seizure propagation to the STP), extent of resection was confirmed with MRI.

\section{Data Collection and Analysis}

The basic clinical information was collected from a prospectively maintained epilepsy surgery database. Additional information was obtained through a retrospective review of the medical records (by Y.N., A.J.S., and A.S.V.), including whether patients experienced seizure-related auditory symptoms, electrode coverage, monitoring results, the extent of seizure focus resection, outcomes, and complications. The seizure outcome was assessed based upon the clinic note from the last known clinic visit at the authors' institution as of February 2018, using the modified Engel score. ${ }^{3}$ The seizure outcome was evaluated only for patients who had a follow-up duration of at least 1 year. The iEEG monitoring records were reviewed and visually inspected (by A.J.S. and M.A.G.). These cases were categorized based upon how quickly the STP became involved from electrographic seizure onset, arbitrarily into within 1 second, between 1 and 15 seconds, after 15 seconds, or not involved. We statistically tested whether existence of seizure onset in the STP was associated with better or worse outcome by using Fisher's exact test, one of the categories being propagation time of seizure in the STP (within 1 second vs after 1 second), and the other category being Engel outcome (I vs II or worse).

\section{Results}

\section{Clinical Demographics}

During the study period, a total of 91 patients underwent craniotomy and placement of intracranial electrodes for iEEG. Fifty-two of these patients (57.1\%) had at least 1 depth electrode placed within the STP (Table 1). Among the patients with STP coverage, an HG depth electrode was used in $46(88.5 \%)$ patients. In $39(75.0 \%)$ patients, the HG depth electrode was the only STP depth electrode placed. In addition to an HG depth electrode, ATO and PT depth electrodes were placed in $2(3.8 \%)$ and $5(9.6 \%)$ patients, respectively. Five (9.6\%) patients had a single STP electrode through the ATO, and 1 (1.9\%) patient had a single electrode placed within the PT. The age of the 52 patients with the STP coverage averaged $34 \pm 11$ years (range 9-56 years). Thirty of these patients were male. 

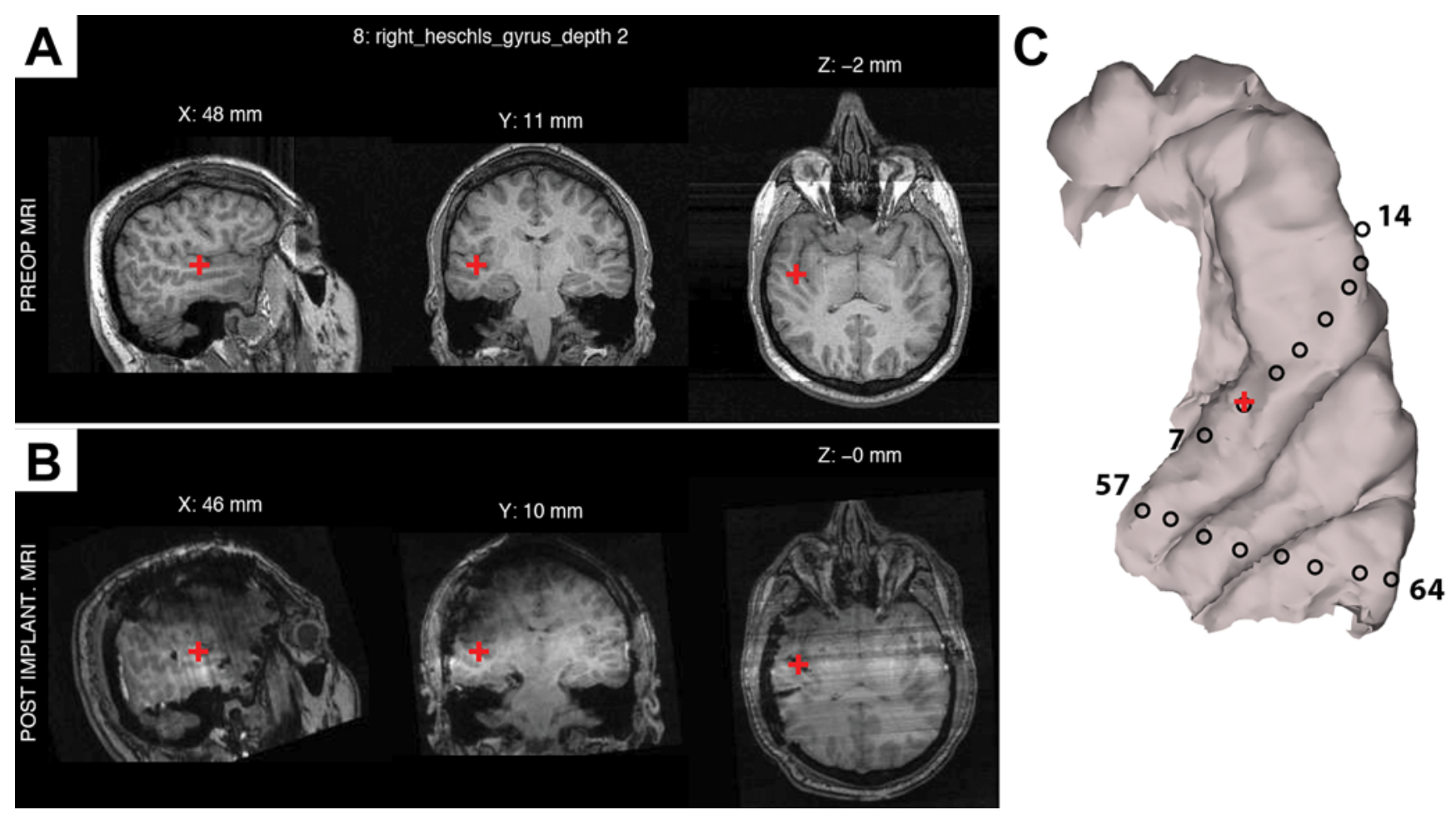

FIG. 2. Preoperative and postimplantation MRI with projection of STP electrodes. A: Sagittal, coronal, and axial T1-weighted preoperative MR images with the location of one of the HG depth electrode contacts mapped (red cross) based on postoperative MRI and CT. B: Sagittal, coronal, and axial T1-weighted MR images acquired after implantation showing the location of the same contact (red cross). C: Top-down view of the right STP showing the locations of the HG (contacts 7-14) and PT (contacts 57-64) depth electrode contacts projected on the surface rendering of the STP. Note that the second deepest contact of the HG depth electrode, marked in $A$ and $B$, is also marked with a red cross in $C$. Figure is available in color online only.

\section{STP Involvement, Auditory Symptoms, and Surgical Outcomes}

The temporal lobe was involved primarily (i.e., seizure onset) or secondarily (i.e., seizure propagation) as part of the presumed epileptogenic zone in 42 cases (Table 2). The mean age was $33.4 \pm 11.4$ years. The number of patients with right, left, and bilateral handedness were 34,7 , and 1 , respectively. A Wada test was performed in 32 cases. The language dominant side that was confirmed with the Wada test was right in 2 cases, left in 27, and bilateral in 3 . The primary seizure-onset zones were localized in the temporal lobe in all but 2 cases. Of the 2 patients who did

TABLE 1. Intracranial EEG cases with coverage of the STP

\begin{tabular}{cc}
\hline STP Coverage & No. of Cases $(\%)$ \\
\hline HG covered & \\
\hline HG only & $39(75.0)$ \\
\hline HG plus ATO & $2(3.8)$ \\
\hline HG plus PT & $5(9.6)$ \\
\hline ATO only & $5(9.6)$ \\
\hline PT only & $1(1.9)$ \\
\hline Total & 52 \\
\hline
\end{tabular}

ATO = anterior temporal operculum; $\mathrm{HG}=$ Heschl's gyrus; $\mathrm{PT}$ = planum temporale; STP = superior temporal plane. not have a primary seizure focus in the temporal lobe, one had a history of prior left-sided ATL and the iEEG recording demonstrated a seizure focus within the left ventral frontal lobe and seizure activity secondarily propagated to the residual mesial temporal lobe. This patient underwent resection of the left ventral frontal cortex and residual mesial temporal structures. The other patient was noted to have diffuse independent seizure foci primarily in the parietal lobe but also the mesial temporal and frontal areas, and this patient did not undergo resective surgery. In 36 (85.7\%) of these cases (with primary or secondary temporal lobe involvement) in this series, the STP was involved at seizure onset (i.e., $0-1$ second) or became involved during seizure propagation (i.e., $1-15$ seconds or $>15$ seconds). Specifically, the STP electrodes became involved within 1 second of seizure onset ("seizure focus") in 5 cases (11.9\%), between 1 and 15 seconds in 5 cases (11.9\%), and after at least 15 seconds in 26 cases (61.9\%). In 6 cases (14.3\%), there was no involvement of the STP electrodes in either seizure onset or subsequent propagation.

In those cases with seizure onset in the STP (Table 3), involvement of STP electrodes preceded involvement of any contacts outside of the temporal lobe. In 2 cases with class II outcome, seizure propagated to the frontal lobe 1 second after onset at the STP; however, in the other 3 cases, seizure activity stayed within the STP for a prolonged time before it spread to other areas. Three of 5 patients who had seizure onset in the STP had lesions in the 
TABLE 2. Presence of auditory symptoms and seizure outcomes depending upon ictal involvement of STP electrodes

\begin{tabular}{|c|c|c|c|c|c|c|c|c|c|c|c|c|c|c|c|}
\hline \multirow{2}{*}{$\begin{array}{l}\text { STP Ictal } \\
\text { Involvement }\end{array}$} & \multirow[b]{2}{*}{ Total } & \multicolumn{3}{|c|}{ MRI Findings } & \multirow[b]{2}{*}{ IID } & \multirow{2}{*}{$\begin{array}{l}\text { Auditory } \\
\text { Sx }\end{array}$} & \multicolumn{5}{|c|}{ Resection } & \multicolumn{4}{|c|}{ Modified Engel Class } \\
\hline & & Normal & MTS & Lesion* & & & All Cases & Cases w/ FU > $1 \mathrm{Yr}$ & ATL & ATL+ & $\mathrm{T} \dagger$ & I & II & III & IV \\
\hline $0-1 \mathrm{sec}$ & 5 & $2(40)$ & $1(20)$ & $3(60)$ & $4(80)$ & 0 & 4 & 4 & 0 & 4 & 0 & $1(25)$ & $3(75)$ & 0 & 0 \\
\hline $1-15$ secs & 5 & $3(60)$ & $1(20)$ & $1(20)$ & $5(100)$ & $1(20)$ & 4 & 3 & 1 & 2 & 0 & $2(67)$ & $1(33)$ & 0 & 0 \\
\hline$>15$ secs & 26 & $8(31)$ & $11(42)$ & $9(35)$ & $7(27)$ & $3(12)$ & 22 & 22 & 14 & 6 & 2 & $18(82)$ & $3(14)$ & $1(5)$ & 0 \\
\hline Not involved & 6 & $1(17)$ & $1(17)$ & $4(67)$ & $1(17)$ & $1(17)$ & 4 & 4 & 2 & 1 & 1 & $4(100)$ & 0 & 0 & 0 \\
\hline Total & 42 & 14 & 14 & 17 & 17 & 5 & 34 & 33 & 17 & 13 & 3 & $25(76)$ & $7(21)$ & $1(3)$ & 0 \\
\hline
\end{tabular}

ATL = anterior temporal lobectomy; ATL+ = ATL plus resection of extensive temporal cortex/lesion or cortex of other lobe; IID = interictal discharges; FU = follow-up; MTS = mesial temporal sclerosis; $\sec (\mathrm{s})=\operatorname{second}(\mathrm{s}) ; \mathrm{STP}=$ supratemporal plane; $\mathrm{Sx}=$ symptoms; $\mathrm{T}=$ temporal.

Values are numbers of cases (\%).

${ }^{*}$ Refers to lesion other than MTS.

$\dagger$ Resection of temporal cortex/lesion without resection of mesial temporal lobe.

temporal lobe; 2 of these 3 patients had undergone ATL without success and 1 had had a perinatal stroke (Table $3)$. In the remaining 2 cases, no evidence of a lesion was seen on MRI (MRI nonlesional cases). MTS, other lesions, and normal MRI were seen in every class of STP seizure involvement (Table 2).

The STP electrode(s) detected interictal discharges in a total of 17 cases $(40.5 \%)$, and this was most often observed in cases in which the STP was noted to be involved in seizure onset or early propagation (e.g., $0-1$ seconds, $1-15$ seconds).

Despite frequent propagation of seizure activity in the STP, where the primary and higher-order auditory areas reside, auditory symptoms had been reported only by 4 (11.1\%) patients with ictal involvement of the STP (Table 2). No patient with ictal involvement of the STP within 1 second of seizure onset had reported auditory symptoms. Among the patients with the STP involved in 1-15 seconds, involved after 15 seconds, and not involved during seizures, auditory symptoms had been reported only by 1 patient, 3 patients, and 1 patient, respectively.

Thirty-four $(81.0 \%)$ of the 42 patients with primary or secondary temporal lobe involvement ultimately underwent resective surgery (Table 2). Of these 34 patients, 1 was lost to follow-up; the remaining 33 patients had at least 1 year of follow-up and their cases were included for descriptive analysis of the surgical outcome. The duration of follow-up in these 33 cases averaged $5.1 \pm 3.0$ years (range 1.1-11.5 years). Overall, 25 (75.8\%) and 7 (21.2\%) patients achieved modified Engel outcome I ("seizure freedom") and II ("favorable outcomes"), respectively. Of the 5 patients in whom seizure activity was seen in the STP within 1 second of seizure onset, 4 underwent tailored temporal lobe resection that included areas of the early seizure propagation in the STP-1 partial resection and 3 complete resections of STP seizure sites (Tables 2 and 3). The extent of the tailored resection in each of these 4 cases was more extensive than the standard ATL. However, despite the tailored resection, only $1(25.0 \%)$ of these 4 patients achieved an Engel class I outcome, while the other 3 patients in the group achieved an Engel class II outcome. The patient with an Engel class I outcome was the only patient of the 4 who did not have interictal discharges involving the STP. Among 3 patients who had seizure propagation to the STP between 1 and 15 seconds and had follow-up duration longer than 1 year, 1 patient had standard ATL, and the remaining 2 patients underwent ATL plus tailored resection (Table 4). Of the patients with seizure propagation to the STP later than 15 seconds and those without STP involvement, 16 patients had ATL, 7 patients had ATL plus tailored resection, and 3 patients underwent resection of the temporal cortex or lesion without resection of the mesial temporal lobe. The groups of patients who had STP propagation 1-15 seconds, after more than 15 seconds, and no involvement achieved Engel class I outcome in $67 \%, 82 \%$, and $100 \%$, respectively. Fisher's exact test showed a significant $(\mathrm{p}<0.05)$ association between seizure onset $(0-1$ second) in the STP and worse outcome (Engel class II or worse).

Secondary seizure foci were detected in 3 of the patients who had seizure onset in the STP, and these patients all had Engel class II outcomes. Among patients who had STP propagation between 1 and 15 seconds, 2 patients had secondary seizure foci. One of them achieved a class I outcome, and the other had a class II outcome. We observed secondary seizure foci in 6 patients who had STP involvement later than 15 seconds after seizure onset. Five of them achieved Engel class I outcome, and the remaining patient had a class II outcome.

\section{Complications}

There were no hemorrhagic complications specifically related to placement of STP electrodes (e.g., intraparenchymal hemorrhage) in this series of 52 patients. No patients experienced subjective deterioration of auditory functions while they had electrodes in the STP or after electrodes were removed. Given the absence of clinically significant auditory symptoms perioperatively, no formal testing of auditory function was performed in any patients in this series.

\section{Discussion}

Temporal lobectomy has been shown to be highly effective in reducing the frequency of seizures in patients with medically intractable TLE..${ }^{15}$ However, in all reported surgical series, a subset of patients did not achieve seizurefree outcomes. In general, strategies that favor more extensive resections in both temporal and extra-temporal lobe epilepsy appear to correlate with higher seizure-free 


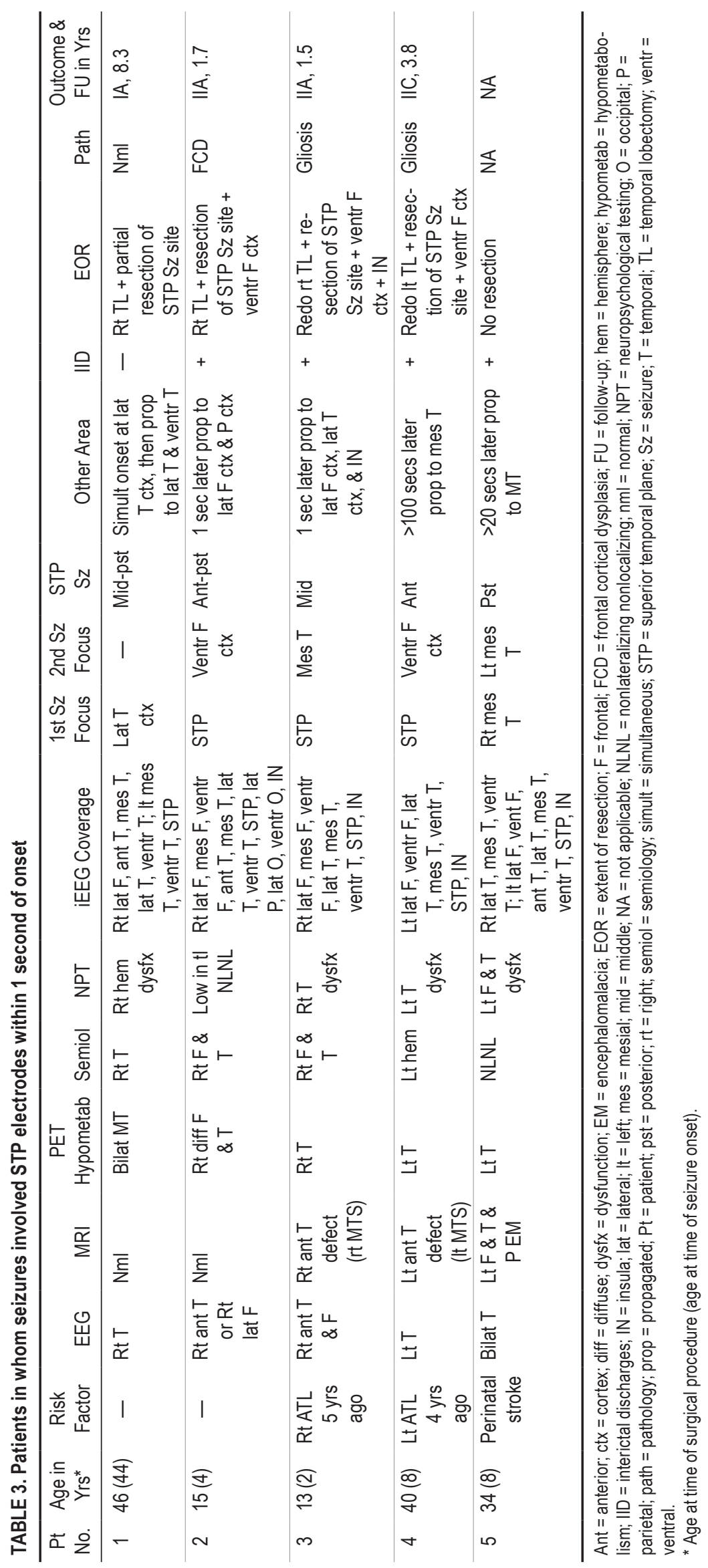




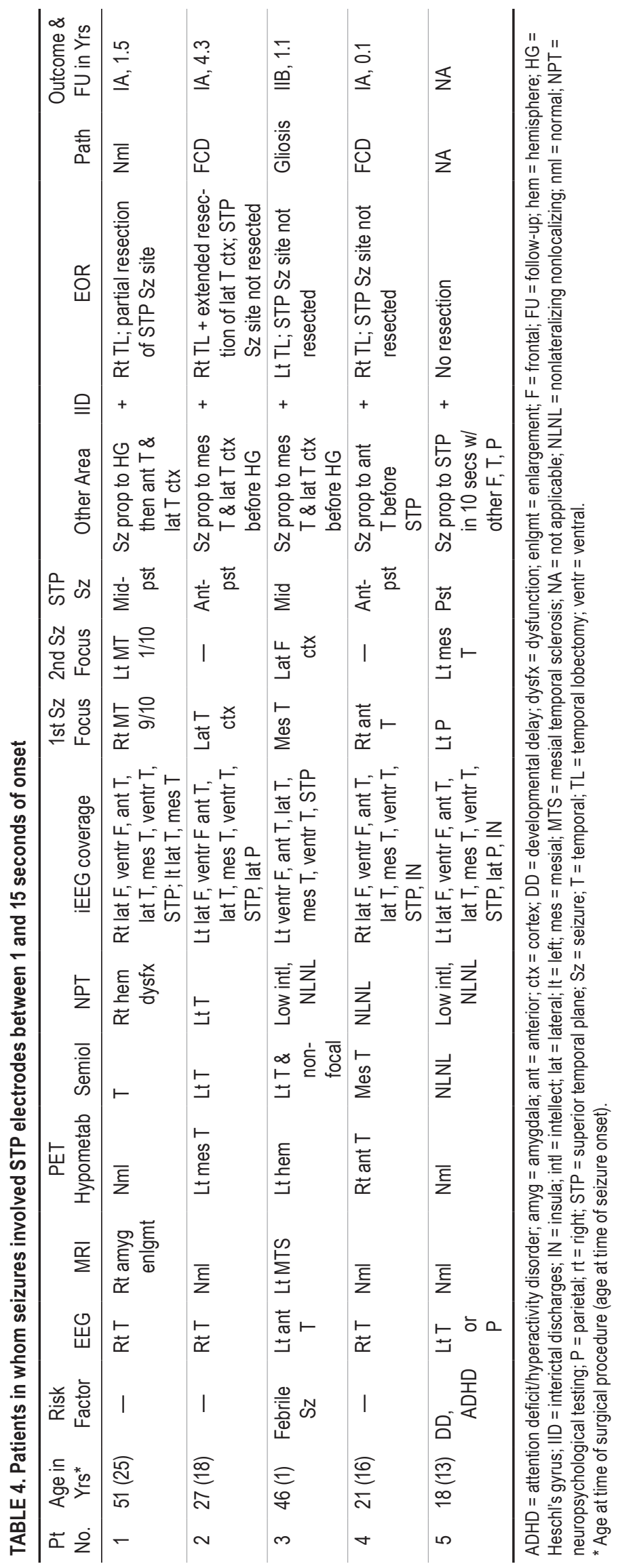


outcome rates, ${ }^{14}$ although the "optimal extent" of resection in TLE remains controversial. ${ }^{13}$ One factor that appears to contribute to suboptimal results following TLE surgery is involvement of brain regions outside the typical extent of TLE surgery as part of a patient's epileptogenic zone (i.e., temporal plus epilepsy). ${ }^{1,12}$ In addition, different areas within the temporal lobe may be differentially involved in seizure onset and propagation, ${ }^{2,8}$ and this may impact the efficacy of resective surgery. Although the mesial temporal structures and the ventral/lateral cortex are readily accessible for invasive recording with depth and subdural surface electrodes and are frequently covered, the dorsal temporal cortex (i.e., STP) presents technical challenges for electrode coverage. At our institution, we use depth electrodes to sample the STP with the intent of obtaining coverage of the dorsal temporal lobe in cases of suspected TLE. In this study, we assessed the utility and safety of placing these STP electrodes.

STP electrodes were frequently involved during seizure onset and propagation in cases in which the temporal lobe was primarily or secondarily involved (85.7\%). Our data showed that involvement of STP electrodes at seizure onset or very early propagation within 1 second had a statistically significant association with worse seizure-free outcome. The rates of Engel class I outcome were 25\%, 67\%, and $82 \%$ for patients with ictal involvement of the STP electrodes within 1 second, in the 1-15 second epoch, and after 15 seconds, respectively. In contrast, all the patients who had no ictal involvement of the STP attained seizure freedom. In addition, interictal discharges were most often observed in patients with seizure patterns that involved rapid progression to the STP. Interictal discharges were detected in all the patients with ictal involvement of the STP within 1 second, except the one patient who achieved seizure freedom. Our findings show that patients with ictal and interictal involvement of the STP have more extensive epileptogenic zones and may have less favorable surgical outcomes, even when the resection is extended to include portions of the STP.

Another significant finding was that there is no predictable relationship between auditory symptoms and the presence or absence of epileptic activity within the STP. Much of the neocortex of the STP is activated by auditory stimuli, but many patients with TLE do not report auditory symptoms associated with their seizures. In addition, auditory symptoms have been more commonly associated with the lateral (neocortical) subtype than the mesial (limbic) or mesial-lateral subtypes of TLE, but many patients with the lateral subtype have no auditory symptoms. ${ }^{8}$ Our finding shows that the absence of auditory symptoms does not rule out involvement of the STP in the seizure network and there may be a variety of possible explanations for this observation.

Within the group of patients who had primary seizure onset in the STP, the 3 patients who had secondary seizure foci had worse outcomes than those who did not. However, in the other groups of patients who had later propagation of seizure in the STP, there were many patients who achieved class I outcome regardless of the presence of secondary seizure foci. Therefore, existence of secondary seizure foci by itself does not appear to relate to seizure free outcome. However, it is unclear whether there is an interaction effect that would make outcome worse in cases with primary seizure onset in the STP and existence of secondary seizure focus outside of the STP.

Electrical activation of many regions of auditory-related STP cortex will not cause a patient to experience an auditory percept. ${ }^{4}$ The regions of auditory cortex that consistently evoke an auditory percept when electronically stimulated occupy a small and highly circumscribed portion of the STP, most often within the posteromedial region of $\mathrm{HG}$ within presumed primary auditory cortex. Another consideration is the neural processing time epochs required for a subject to describe a sensory percept induced by electrical activation of sensory cortex. When electrical stimuli are delivered to somatosensory cortex, the pulse train duration must exceed approximately 500 msec before patients reliably report somatosensory symptoms, although greater stimulation intensity can lower this time. ${ }^{7}$ We have observed that pulse train durations above $150 \mathrm{msec}$ can evoke an auditory percept when electrically stimulating primary auditory cortex. The subsequent neural processing that takes place in nonsensory brain regions that subserve the functions that enable a patient to remember and report a percept presumably extends over significantly longer time periods. When seizure activity involving the STP propagates through the seizure network, a patient's level of consciousness and recall capacity can become impaired. When this propagation occurs with sufficient speed, the neural processes required for perception and reporting of auditory percepts may be disrupted. For these reasons, it is valuable to place recording electrodes within the STP to provide dorsal temporal lobe coverage and define the distributed seizure network in patients with TLE even in the absence of auditory symptoms.

There were no complications related to placement of the STP depth electrodes in the 52 cases. This finding confirms the safety of this method whereby frameless stereotactic techniques are used to insert electrodes by means of an entry point on the lateral surface of the superior temporal gyrus and trajectories that avoid STP sulci and blood vessels with the sylvian fissure. The diagnostic information obtained was useful in delineating seizure networks that frequently involve the STP even in patients without auditory symptoms. STP electrode data proved useful prognostically in that patients with early involvement of the STP after seizure onset had lower seizure-free outcome rates following resective surgery.

One limitation of our approach is the sparse area of STP coverage achieved when only a single electrode is placed in this region. There may have been undetected seizure involvement of the STP in no-involvement cases or earlier STP involvement in later-involvement cases. We are also unable to predict what the seizure control outcomes might have been if we had not removed the STP seizure sites. Our strategy appears to be effective in identifying patients who have involvement of the STP in their seizure network, but the coverage may be insufficient to specifically localize STP involvement to the degree necessary to guide effective resections of the dorsal temporal lobe in this subset of patients. Given the demonstrated safety of the STP electrode placement method, we can now address 
this concern by developing electrode coverage strategies directed at achieving enhanced areal coverage of the dorsal temporal lobe.

Another limitation of this study was the lack of a routine postoperative high-resolution MRI study to objectively evaluate the extent of resection in each case. As resective surgery involved standard ATL in most of the cases in this study, only a brain CT study without contrast was routinely obtained to rule out postoperative complications. The lack of postoperative high-resolution MRI studies precluded the detailed analysis of the relationship among resection extent, the ictal/interictal involvement of the STP, and seizure outcomes.

This study showed a possible critical role of the STP in TLE and the utility of STP electrodes in providing useful information of prognostic values. We were unable to identify factors that would predict existence of seizure foci in the STP from preoperative epilepsy workup data. Therefore, it may be reasonable to routinely place such electrodes in all suspected TLE cases regardless of the presence of auditory symptoms. However, whether such electrodes actually help improve seizure outcomes remains to be answered, given that tailored more extensive resection involving portions of the STP in a subset of the patients did not necessarily improve outcomes in this study.

\section{Conclusions}

Depth electrodes can be placed safely within the STP, and the recordings obtained from these electrodes provide valuable information about the seizure network in patients with TLE. The STP was involved in the seizure network of the majority of TLE patients in this case series, and most of these patients had no auditory symptoms. Early involvement of the STP in the seizure propagation pattern may lead to worse surgical outcomes, and the information gained from studying this region may potentially be useful in preoperative counseling and surgical planning. Further methodologic refinements are needed to provide enhanced coverage of the STP that may facilitate more effective resection strategies in this subset of patients.

\section{References}

1. Barba C, Rheims S, Minotti L, Guénot M, Hoffmann D, Chabardès $\mathrm{S}$, et al: Temporal plus epilepsy is a major determinant of temporal lobe surgery failures. Brain 139:444451, 2016

2. Bartolomei F, Cosandier-Rimele D, McGonigal A, Aubert S, Régis J, Gavaret M, et al: From mesial temporal lobe to temporoperisylvian seizures: a quantified study of temporal lobe seizure networks. Epilepsia 51:2147-2158, 2010

3. Engel JJ, Van Ness PC, Rasmussen TB, Ojemann LM: Outcome with respect to epileptic seizures, in Engel JJ (ed): Surgical Treatment of the Epilepsies, ed 2. New York: Raven Press, 1993, pp 609-621

4. Fenoy AJ, Severson MA, Volkov IO, Brugge JF, Howard MA III: Hearing suppression induced by electrical stimulation of human auditory cortex. Brain Res 1118:75-83, 2006
5. Isnard J, Guénot M, Ostrowsky K, Sindou M, Mauguière F: The role of the insular cortex in temporal lobe epilepsy. Ann Neurol 48:614-623, 2000

6. Isnard J, Guénot M, Sindou M, Mauguière F: Clinical manifestations of insular lobe seizures: a stereo-electroencephalographic study. Epilepsia 45:1079-1090, 2004

7. Libet B, Alberts WW, Wright EW Jr, Delattre LD, Levin G, Feinstein B: Production of threshold levels of conscious sensation by electrical stimulation of human somatosensory cortex. J Neurophysiol 27:546-578, 1964

8. Maillard L, Vignal JP, Gavaret M, Guye M, Biraben A, McGonigal A, et al: Semiologic and electrophysiologic correlations in temporal lobe seizure subtypes. Epilepsia 45:15901599,2004

9. Munari C: Depth electrode implantation at Hopital Sainte Anne, Paris, in Engel JJ (ed): Surgical Treatment of the Epilepsies. New York: Raven Press, 1987, pp 583-588

10. Nagahama Y, Kovach CK, Ciliberto M, Joshi C, Rhone AE, Vesole A, et al: Localization of musicogenic epilepsy to Heschl's gyrus and superior temporal plane: case report. J Neurosurg 129:157-164, 2018

11. Reddy CG, Dahdaleh NS, Albert G, Chen F, Hansen D, Nourski K, et al: A method for placing Heschl gyrus depth electrodes. J Neurosurg 112:1301-1307, 2010

12. Ryvlin P, Kahane P: The hidden causes of surgery-resistant temporal lobe epilepsy: extratemporal or temporal plus? Curr Opin Neurol 18:125-127, 2005

13. Schramm J: Temporal lobe epilepsy surgery and the quest for optimal extent of resection: a review. Epilepsia 49:12961307, 2008

14. Tonini C, Beghi E, Berg AT, Bogliun G, Giordano L, Newton RW, et al: Predictors of epilepsy surgery outcome: a metaanalysis. Epilepsy Res 62:75-87, 2004

15. Wiebe S, Blume WT, Girvin JP, Eliasziw M: A randomized, controlled trial of surgery for temporal-lobe epilepsy. $\mathbf{N ~ E n g l ~}$ J Med 345:311-318, 2001

16. Yvert B, Fischer C, Bertrand O, Pernier J: Localization of human supratemporal auditory areas from intracerebral auditory evoked potentials using distributed source models. Neuroimage 28:140-153, 2005

\section{Disclosures}

The authors report no conflict of interest concerning the materials or methods used in this study or the findings specified in this paper.

\section{Author Contributions}

Conception and design: Nagahama, Dlouhy, Granner, Howard, Kawasaki. Acquisition of data: Nagahama, Schmitt, Vesole, Kovach, Granner. Analysis and interpretation of data: Nagahama, Schmitt, Nakagawa, Granner, Howard, Kawasaki. Drafting the article: Nagahama. Critically revising the article: Nagahama, Schmitt, Dlouhy, Vesole, Gander, Kovach, Granner, Howard, Kawasaki. Reviewed submitted version of manuscript: all authors. Approved the final version of the manuscript on behalf of all authors: Nagahama. Statistical analysis: Nakagawa. Study supervision: Howard, Kawasaki.

\section{Correspondence}

Yasunori Nagahama: University of Iowa Hospitals and Clinics, Iowa City, IA. yasunori-nagahama@uiowa.edu. 